\title{
PERCEPCIÓN DE LOS JÓVENES SOBRE LOS ESPACIOS Y MOMENTOS EN SU PROCESO DE EMPODERAMIENTO. UNA APROXIMACIÓN CUANTITATIVA'
}

\author{
YOUTH PERCEPTION ON SPACES AND MOMENTS IN THE PROCESS \\ OF EMPOWERMENT. A QUANTITATIVE APPROACH \\ PERCEÇÃO DOS JOVENS SOBRE OS ESPAÇOS E MOMENTOS NO SEU \\ PROCESSO DE CAPACITAÇÃO. UMA APROXIMAÇÃO QUANTITATIVA
}

\author{
Ingrid AGUD-MORELL*, Anna CIRASO-CALI'*, Pilar PINEDA-HERRERO* \\ \& Pere SOLER-MASÓ** \\ *Universitat Autònoma de Barcelona, ${ }^{* *}$ Universitat de Girona
}

\begin{tabular}{|c|c|}
\hline & $\begin{array}{r}\text { Fecha de recepción del artículo: } 27.11 .2017 \\
\text { Fecha de revisión del artículo: 6.11.2017 } \\
\text { Fecha de aceptación final: 3.IV.2017 }\end{array}$ \\
\hline $\begin{array}{l}\text { PALABRAS CLAVES: } \\
\text { empoderamiento } \\
\text { juvenil } \\
\text { espacios } \\
\text { empoderadores } \\
\text { incidentes críticos } \\
\text { etapas vitales }\end{array}$ & $\begin{array}{l}\text { RESUMEN: La voz de los jóvenes no ha sido tenida en cuenta en la conceptualización del } \\
\text { empoderamiento y los estudios sobre éste. El presente artículo contribuye a la investiga- } \\
\text { ción de espacios y momentos clave en el proceso de empoderamiento juvenil desde la } \\
\text { propia vivencia en primera persona de } 890 \text { jóvenes. Mediante una aproximación cuanti- } \\
\text { tativa se estudia cómo diferentes espacios de la vida cotidiana contribuyen al desarrollo } \\
\text { de capacidades y cómo éstos permiten aplicarlas. Se identifican vivencias que los jóvenes } \\
\text { perciben como altamente empoderadoras y se estudia la relación del empoderamiento con } \\
\text { las diferentes etapas vitales. Los resultados propician evidencias y contribuyen al debate } \\
\text { y la reflexión sobre el empoderamiento juvenil tanto para las investigaciones futuras como } \\
\text { para optimizar las intervenciones en el trabajo con jóvenes y en el diseño e implementación } \\
\text { de políticas de juventud. }\end{array}$ \\
\hline
\end{tabular}

CONTACTAR CON LOS AUTORES: Ingrid Agud-Morell. Departamento Pedagogía Sistemática y Social. Universidad Autónoma de Barcelona. Edifici G6. Campus de la UAB, 08193 Bellaterra (Cerdanyola del Vallès). ingrid.agud@uab.cat.

FINANCIACIÓN: Proyecto HEBE El empoderamiento de los jóvenes: análisis de los momentos, espacios y procesos que contribuyen al empoderamiento juvenil. MINECO - PROGRAMA ESTATAL DE I+D+| RETOS DE LA SOCIEDAD 2013. Ref.: EDU2013-42979-R. 
KEY WORDS:

youth empowerment empowering spaces critical incidents life stages
ABSTRACT: The voice of youth has not been taken into account in the conceptualization of empowerment and related research. The contents of this article contribute to the study of spaces and key moments in the process of youth empowerment through first-person experiences of 890 young. A quantitative approach will be used in order to consider the contribution of specific ambits of everyday life to capacity development and its application. Personal experiences perceived by youth as highly empowering are identified, and the relationship between empowerment and different life stages is studied. The findings suggest that the spaces generating more youth empowerment are formal and non-formal education settings and work environment. The importance of emancipation and life experiences entailing personal overcoming is also illustrated. In essence, the outcomes provide evidence and contribute to the debate and reflection of youth empowerment so as to promote future research and improve youth work interventions, as well as the design and implementation of youth policies.

PALAVRAS-CHAVE: capacitação juvenil espaços capacitantes incidentes críticos etapas vitais
RESUMO: A voz dos jovens não foi tida em conta na conceptualização da capacitação e nos estudos sobre esta. $O$ presente artigo contribui para a investigação de espaços e momentos-chave no processo de capacitação juvenil a partir da própria vivência na primeira pessoa de 890 jovens. Através de uma aproximação quantitativa, estuda-se como diferentes espaços da vida quotidiana contribuem para o desenvolvimento de competências e como estes permitem aplicá-las. Identificam-se vivências que os jovens percebem como altamente capacitantes e estuda-se a relação da capacitação com as diferentes etapas vitais. Os resultados apontam para o facto de os espaços onde os jovens mais se capacitam serem os da educação formal e não formal e os ambientes laborais. Também visualizam a importância da emancipação e as experiências vitais que requerem uma atitude de superação pessoal. Por fim, os resultados propiciam evidências e contribuem para o debate e a reflexão sobre a capacitação juvenil, tanto para as investigações futuras como para otimizar as intervenções no trabalho com jovens e na conceção e implementação de políticas de juventude.

\section{Introducción}

La diversidad de concepciones sobre el empoderamiento complica los intentos de estudiarlo, medirlo o clasificarlo, de modo que las investigaciones con este propósito (Holte-McKenzie, Forde and Theobald, 2006; Morrissey, 2000; Pearrow, 2011; Peterson, 2014; Pick et al., 2007; Tromp, 2007, Wright, 2010) no consiguen, tal como afirma Mosedale (2003), un método aceptado ni para su medición ni para el seguimiento de los cambios que con él se producen. Wagaman (2011) en este mismo sentido concluye que cada definición de empoderamiento tiene su método particular de medirlo. Hay también otras limitaciones a considerar como el hecho que la mayoría de investigaciones se basan en fuentes secundarias o en planteamientos teóricos sin evidencias empíricas (Hennink et al., 2012; Peterson, 2014).

Si centramos la atención en el empoderamiento juvenil, Morton y Montgomery (2013) identifican también la ausencia de investigaciones de calidad que aporten evidencias sobre los resultados de programas de empoderamiento juvenil. Insisten en la importancia de investigar el impacto de los programas de empoderamiento juvenil para entender mejor el valor de éstos en contextos con recursos económicos limitados. La investigación que aquí presentamos pretende contribuir de algún modo en esta dirección, profundizando en los espacios y los momentos en los que el empoderamiento es más reconocido, identificado o puede ser más determinante.

Partimos de un concepto de empoderamiento fundamentado en el análisis sistemático de las definiciones e investigaciones desarrolladas, en los últimos 15 años, sobre el empoderamiento y sobre la manera en qué ha sido conceptualizado y aplicado en el ámbito juvenil (Úcar, et al., 2016). El resultado de esta investigación nos permite ensayar una propuesta de concepto de empoderamiento a partir del cual construimos una aproximación de modelo educativo (Soler et. al, 2017). El trabajo realizado propone una triple mirada a partir de las dimensiones de espacios, momentos y procesos característicos en el empoderamiento de los jóvenes.

El concepto de empoderamiento que manejamos se concreta como el proceso mediante el cual se incrementan las posibilidades de que una persona joven pueda decidir y actuar de forma consecuente sobre todo aquello que afecta a su propia vida; y participar en la toma de decisiones e intervenir de forma compartida y responsable en lo que afecta a la colectividad de la que forma parte (Soler et. al; 2017).

En los trabajos citados ya avanzamos nuestro posicionamiento en relación a dos dimensiones fundamentales a la hora de analizar el empoderamiento. La primera se refiere a las capacidades personales y a los medios a través de los cuales es posible adquirirlas, desarrollarlas o ponerlas en juego. Se trata de una dimensión directamente 
relacionada con la trayectoria vital de las personas y con sus procesos de aprendizaje y formación. La segunda se refiere al entorno y a las posibilidades y oportunidades de acción que éste ofrece o deniega. Se interpreta, en este sentido, que el medio o el contexto en el que las personas, los grupos y las comunidades desenvuelven su vida puede ser posibilitadores o inhibidores de las decisiones y acciones que éstas puedan tomar. Se trata de la posibilidad de llevar a cabo acciones de manera consecuente con las decisiones tomadas sobre todo aquello que les afecta. Así, no sólo se trata de tener acceso a los recursos sino de lograr capacidad de decisión sobre ellos (Sathar and Kazi, 1997; Kishor, 1999 [citado en Pick et al. 2007]).

Fetterman y Wandersman (2007) advierten que nadie empodera a nadie, sino que se trata de un proceso intransferible que debe realizar cada uno o la comunidad misma. También Boluijt \& Graaf (2010) coinciden en esta dirección cuando concluyen que la idea que ha estado presente en el desarrollo del empoderamiento es precisamente ayudar a las personas a ayudarse a sí mismas. Esta afirmación nos invita a poner en valor la importancia de los contextos, los espacios y los ambientes en los que se mueven y crecen los jóvenes. El conocimiento de ambientes y entornos de aprendizaje y experimentación, así como los momentos en los que éstos se identifican o viven con especial intensidad, pueden ser claros determinantes para facilitar el empoderamiento. Jennings et al. (2008) insisten en esta idea al proponer como estrategia para el empoderamiento juvenil la creación de oportunidades y el planteamiento de retos en ambientes acogedores y seguros. McHale, Dotterer y Kim (2009) también abordan el análisis de los ambientes o espacios de empoderamiento juvenil a partir del estudio de las actividades diarias y de vida cotidiana en espacios rutinarios y habituales. Esta dimensión es importante porque contempla desde los contextos más próximos o cercanos (familia, grupo de iguales, etc.), hasta los contextos más macro, como pueden ser la política, la economía o la cultura. Otros autores, como Kelly \& Carson (2012) y Nolas (2014), incluyen en estos espacios las asociaciones juveniles y los espacios de participación como contextos que potencian el empoderamiento. También Masa (2009) propone el análisis de diferentes ambientes de empoderamiento: familiar y de convivencia; económico; grupo de iguales; ocio y tiempo libre; emocional, afectivo y relaciones sexuales $y$, finalmente, proyecto de vida. Todo ello no deja de ser una clasificación de posibles espacios de empoderamiento de los jóvenes. Por su parte, Stolte et al. (2014) profundizan en la idea de los ambientes de empoderamiento juvenil y proponen en este caso rúbricas para promover estos ambientes.

Russell et al. (2009) advierten que el empoderamiento es un término popular en el campo de los programas de desarrollo de la juventud (Huebner, 1998), aunque según estos autores los estudios sobre empoderamiento juvenil desdibujan el concepto haciéndolo equivalente a "liderazgo juvenil”, “implicación cívica", "auto-eficacia” o “activismo juvenil". Los estudios existentes ponen de relieve algunas diferencias entre el empoderamiento de jóvenes y de adultos (Peterson et al., 2011) y sobre el papel que los adultos deben ejercer (Blanchet-Cohen y Brunson, 2014). Se afirma también que tanto la investigación teórica como la empírica sobre el empoderamiento se ha llevado a cabo fundamentalmente en el ámbito de los adultos. Hay pues, la idea de un sesgo adulto céntrico que invita a un estudio más profundo, detallado y comparado de las formas en las que el empoderamiento se produce en los jóvenes (Kaplan et al., 2009). En este caso, la investigación que presentamos parte de la opinión y valoración directa de los mismos jóvenes a partir de un amplio cuestionario precisamente sobre los espacios y momentos de empoderamiento juvenil. En la investigación que aquí presentamos, se hace particular énfasis en la inclusión de la percepción e interpretación de los mismos jóvenes en el estudio de los espacios y momentos de empoderamiento, salvando así el sesgo al que hacen referencia los autores citados.

Objetivos:

El estudio tiene el propósito de explorar el proceso de empoderamiento juvenil tomando como principal fuente de información la percepción subjetiva de los jóvenes. Por ello nos proponemos los siguientes objetivos específicos:

1) Identificar los espacios en los que se produce el empoderamiento de los jóvenes.

2) Indagar en qué medida los incidentes críticos contribuyen al empoderamiento juvenil.

3) Conocer las etapas de la vida de los jóvenes en las que el empoderamiento se da con mayor intensidad.

La finalidad última del estudio es aportar datos proporcionados por los propios jóvenes, propiciar evidencias y contribuir al debate y la reflexión sobre el empoderamiento juvenil, tanto para las investigaciones futuras como para optimizar las intervenciones en el trabajo con jóvenes y en el diseño e implementación de políticas de juventud. 


\section{Metodología}

La investigación que aquí se presenta es un estudio sincrónico que recoge las características de un grupo de jóvenes, en un contexto específico, Cataluña; y en una situación significativa debido a las condiciones socio-económicas que actualmente impactan negativamente en la vida de los jóvenes en términos de acceso a la educación, a la vivienda y al mundo laboral. Circunstancias en las cuales el empoderamiento se convierte en un concepto especialmente relevante.

Nuestra aproximación metodológica es de carácter cuantitativo, inspirado por el paradigma neopositivista. Hemos partido de un planteamiento deductivo para la comprobación empírica de la teoría formulada previamente. En este tipo de aproximación, ha sido de gran importancia el análisis de la literatura existente así como estudios cualitativos previos del mismo proyecto de investigación. Esta aproximación nos permite una observación científica, una recogida de datos objetivos y estandarizados (Corbetta, 2010).

Además nos ha permitido acceder a la subjetividad de un número elevado de jóvenes: la recogida de datos se produce a nivel individual pero el interés radica en el resultado conjunto de la muestra para caracterizar al colectivo.

En relación a la muestra, ésta es de carácter intencional, guiado por criterios de accesibilidad y de inclusión de jóvenes participantes con diferentes perfiles. El tamaño inicial de muestra fue de 1067 informantes de los cuales, tras la depuración de valores extremos y respuestas incoherentes quedó una muestra final de $\mathrm{N}=890$ jóvenes con edades de 19 a 25 años (en media de 22 años, desviación típica 1.67). Para una muestra aleatoria equivalente, se trataría de una muestra representativa de los jóvenes catalanes de esta franja de edad (población 535.706, según el Censo de Población y Viviendas 2011 del INE), con un margen de error del 3,3\% y un nivel de confianza de 95\%. La descripción detallada de la muestra se expone en la tabla 1.

\begin{tabular}{|c|c|}
\hline \multicolumn{2}{|r|}{ Tabla 1: Descripción de la muestra } \\
\hline Ítems & $\%$ \\
\hline Sexo & 66,5\% Mujeres; 33,5\% Hombres. \\
\hline Provincia & 68,9\% Barcelona; 16,5\% Girona; 5,2\% Lleida; 9,4\% Tarragona. \\
\hline Entorno de residencia & 77,6\% Urbano; $22,4 \%$ Rural. \\
\hline Lugar de nacimiento & $\begin{array}{l}\text { 81,8\% Cataluña; 22,4\% Europa; 5,5\% Latinoamérica; 4,8\% África; 4,5\% resto de España; } \\
\text { o,8\% Asia; O,1\% Norteamérica. }\end{array}$ \\
\hline Nivel de estudios finalizados & $\begin{array}{l}\text { 39,3\% Bachillerato; } 19,6 \% \text { Ciclo formativo de grado superior; } 14,6 \% \text { Diplomatura, licenciatura } \\
\text { o grado; } 9,4 \% \text { Educación secundaria obligatoria; 6,8\% Ciclo formativo de grado medio; } \\
4,4 \% \text { Educación primaria o inferior; } 4 \% \text { Posgrado o máster y } 2 \% \text { Programas de garantía social, } \\
\text { cualificación inicial o escuela de adultos. }\end{array}$ \\
\hline Trabajo & $\begin{array}{l}57,5 \% \text { No; } 42,5 \% \text { Sí. Entre los jóvenes que trabajan: } 64.3 \% \text { no viven principalmente de sus } \\
\text { ingresos. }\end{array}$ \\
\hline Situación de convivencia & $\begin{array}{l}\text { 74,6\% Con la familia de origen; } 15,9 \% \text { En piso compartido; 6,5\% En pareja; 2,4\% Solo; } \\
\text { 0,7\% Residencia de estudiantes. }\end{array}$ \\
\hline
\end{tabular}

Para la construcción del instrumento, la tarea clave ha sido la de operacionalizar los conceptos constitutivos de la teoría, es decir su transformación en variables observables. El proceso de operacionalización, además de tener en cuenta la literatura existente, se sustentó en la fase de investigación previa a este estudio, donde mediante una técnica lexicométrica se identificó la representación conceptual que tienen tanto jóvenes como profesionales de juventud sobre el empoderamiento en relación con sus espacios, momentos y procesos (Pineda, Agud y Núñez, 2015). El contenido del instrumento ha sido validado por un comité de expertos formado por 20 investigadores y 15 profesionales de juventud; y la fiabilidad en cuanto a consistencia interna resulta en un Alfa de Cronbach de $792(\mathrm{~N}=890)$ para las escalas.

Se trata de un cuestionario que recoge en primer lugar datos detallados del perfil de los jóvenes, a nivel socio-económico, educativo, laboral, familiar, de emancipación y de participación social; para luego indagar sobre cómo han contribuido sus 
diferentes experiencias vitales en su proceso de empoderamiento.

Las dimensiones que conforman el instrumento son: (1) Vivencias importantes o momentos críticos vitales: el objetivo es identificar los que hayan acontecido en la vida del joven e indicar el grado en que los aprendizajes realizados en la vivencia han sido relevantes para su vida y el grado en que la vivencia le ha llevado a tomar autónomamente decisiones importantes sobre su vida. (2) Espacios: con el objetivo de identificar espacios que hayan contribuido al desarrollo de sus capacidades y el grado que les ha permitido dicho espacio aplicar esas capacidades. (3) Etapas vitales: con el objetivo de señalar para cada etapa vital el grado en que ha tenido capacidades para actuar sobre su realidad y el grado en que se ha podido aplicar esta capacidad para actuar sobre su realidad. Cada dimensión lista una serie de variables configuradas a partir de las acciones anteriormente mencionadas. Por cada ítem de la lista, los jóvenes podían valorar -en una escala de intensidad del 1 (nada) al 4 (mucho)- su propio empoderamiento usando las dos dimensiones que según nuestro posicionamiento teórico componen este concepto (Úcar et al. 2016): (E1) desarrollo de capacidades para actuar autónomamente y (E2) aplicación de esas capacidades para actuar sobre su propia vida.

El cuestionario se administró tanto en formato papel como online (usando la plataforma SurveyMonkey) entre julio y diciembre de 2015. Contamos con la colaboración de profesionales e instituciones trabajando en el ámbito de la juventud que posibilitaron la participación de una diversidad de jóvenes con diferente clase social, nivel de estudios, perfiles profesionales y de diferentes residencias de origen.

El objeto del análisis en esta aproximación ha sido explicar estadísticamente la variación en los valores de las variables en función de las variables dependientes en los sujetos. Para dar respuesta a los objetivos anteriormente mencionados se han realizado análisis estadísticos descriptivos y pruebas bivariantes como coeficientes de correlación no paramétrica rho de Spearman y pruebas chi cuadrado con análisis de residuos tipificados corregidos de Haberman (1973).

Esta metodología nos ofrece nueva información sobre el empoderamiento, aun así presenta algunas limitaciones que apuntan a líneas futuras de investigación, tales como la introducción de estrategias cualitativas para ahondar en los resultados o la aplicación de un estudio longitudinal que permitiera un seguimiento en la trayectoria de los jóvenes.

\section{Resultados}

Presentaremos a continuación los hallazgos más significativos de este estudio en relación a los objetivos específicos que nos proponíamos alcanzar:

(1) Identificar los espacios en los que se produce el empoderamiento de los jóvenes.

A continuación presentamos los resultados descriptivos (tabla 2) en relación a los espacios teóricos de empoderamiento juvenil. Cada dimensión corresponde a un espacio de empoderamiento. Ésta agrupación permite realizar un análisis más completo relacionando entornos similares. Para cada espacio concreto dentro de cada dimensión, exponemos el número de individuos que ha formado parte del espacio en cuestión. La siguiente columna muestra la media (en una escala de intensidad del 1 -nada- al 4 mucho-) en que ese espacio se considera como contribuyente al desarrollo de las capacidades para actuar autónomamente sobre su realidad (E1) y la última columna indica la media para el espacio en la medida que éste permite aplicar las capacidades (E2).

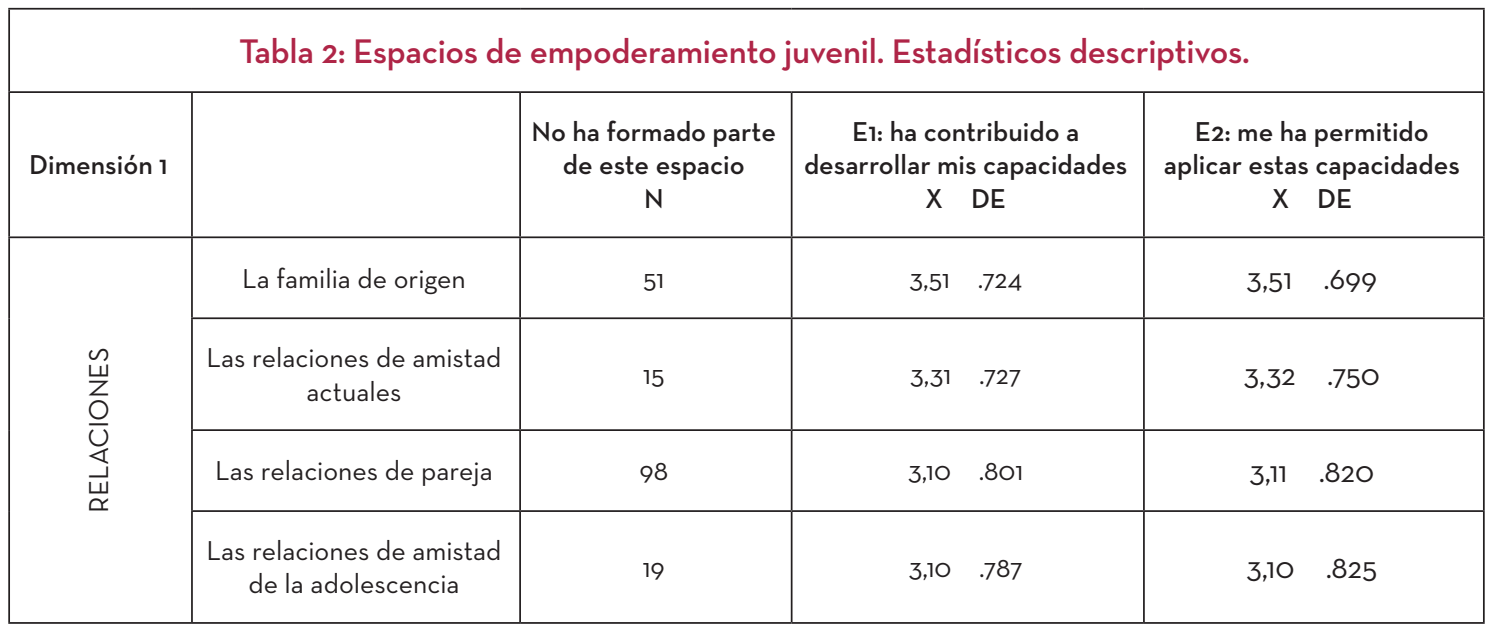




\begin{tabular}{|c|c|c|c|c|c|c|}
\hline Dimensión 2 & & $\begin{array}{c}\text { No ha formado parte } \\
\text { de este espacio } \\
\mathrm{N}\end{array}$ & $\begin{array}{r}\text { E1: ha con } \\
\text { desarrollar mi } \\
X\end{array}$ & $\begin{array}{l}\text { tribuido a } \\
\text { s capacidades } \\
\text { DE }\end{array}$ & $\begin{array}{r}\text { E2: me ha } \\
\text { aplicar estas } \\
\mathrm{X}\end{array}$ & $\begin{array}{l}\text { permitido } \\
\text { capacidades } \\
\text { DE }\end{array}$ \\
\hline \multirow{6}{*}{ 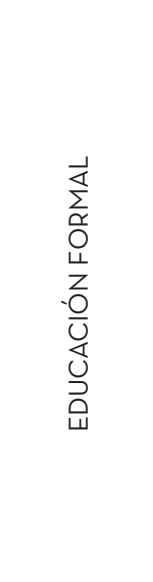 } & $\begin{array}{c}\text { Educación superior } \\
\text { (estudios universitarios) }\end{array}$ & 232 & 3,37 & .744 & 3,34 & .775 \\
\hline & $\begin{array}{c}\text { Educación secundaria } \\
\text { post-obligatoria } \\
\text { (Bachillerato y FP) }\end{array}$ & 104 & 3,18 & .806 & 3,16 & .830 \\
\hline & Formación ocupacional & 572 & 3,03 & .912 & 3,08 & .914 \\
\hline & Escuela de adultos & 692 & 2,85 & .993 & 2,85 & 1.05 \\
\hline & $\begin{array}{c}\text { Educación infantil y } \\
\text { primaria }\end{array}$ & 27 & 2,93 & .864 & 2,87 & .876 \\
\hline & $\begin{array}{c}\text { Educación secundaria } \\
\text { obligatoria (ESO) }\end{array}$ & 34 & 2,85 & .879 & 2,81 & .878 \\
\hline
\end{tabular}

\begin{tabular}{|c|c|c|c|c|}
\hline \multirow{2}{*}{ Dimensión 3 } & $\begin{array}{c}\text { No ha formado parte } \\
\text { de este espacio } \\
\text { N }\end{array}$ & $\begin{array}{c}\text { E1: ha contribuido a } \\
\text { desarrollar mis capacidades } \\
\text { X DE }\end{array}$ & $\begin{array}{c}\text { E2: me ha permitido } \\
\text { aplicar estas capacidades } \\
\text { X } \\
\text { DE }\end{array}$ \\
\hline \multirow{2}{*}{\begin{tabular}{c} 
d \\
\cline { 2 - 5 }
\end{tabular}} & Trabajos estables & 242 & $3,25.819$ & $3,19.875$ \\
\cline { 2 - 6 } & Trabajos temporales & 461 & $3,18.866$ & $3,20.903$ \\
\hline
\end{tabular}

\begin{tabular}{|c|c|c|c|c|c|c|}
\hline Dimensión 4 & & $\begin{array}{c}\text { No ha formado parte } \\
\text { de este espacio } \\
\mathrm{N}\end{array}$ & $\begin{array}{r}\text { E1: ha con } \\
\text { desarrollar mi } \\
X\end{array}$ & $\begin{array}{l}\text { tribuido a } \\
\text { s capacidades } \\
\text { DE }\end{array}$ & $\begin{array}{r}\text { E2: me ha } \\
\text { aplicar estas } \\
\mathrm{X}\end{array}$ & $\begin{array}{l}\text { oermitido } \\
\text { capacidades } \\
\text { DE }\end{array}$ \\
\hline \multirow{8}{*}{ 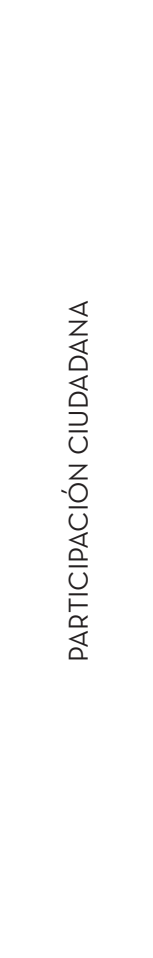 } & $\begin{array}{c}\text { Grupos o asociaciones de } \\
\text { tiempo libre }\end{array}$ & 323 & 3,32 & .830 & 3,34 & .828 \\
\hline & $\begin{array}{c}\text { Asociaciones de tipo } \\
\text { cultural }\end{array}$ & 414 & 3,01 & .902 & 3,02 & .918 \\
\hline & $\begin{array}{c}\text { Asociaciones o } \\
\text { organizaciones de tipo } \\
\text { deportivo }\end{array}$ & 422 & 3,00 & .845 & 2,98 & .912 \\
\hline & $\begin{array}{c}\text { ONG's u otras } \\
\text { asociaciones de carácter } \\
\text { cívico }\end{array}$ & 547 & 2,92 & .932 & 3,02 & .976 \\
\hline & $\begin{array}{c}\text { Consejos de participación } \\
\text { infantil o juvenil }\end{array}$ & 615 & 2,80 & .966 & 2,87 & .994 \\
\hline & $\begin{array}{c}\text { Partidos o organizaciones } \\
\text { de carácter político }\end{array}$ & 613 & 2,35 & 1.01 & 2,29 & 1.05 \\
\hline & $\begin{array}{c}\text { Asociaciones o } \\
\text { confesiones religiosas }\end{array}$ & 656 & 2,31 & 1.18 & 2,29 & 1.19 \\
\hline & $\begin{array}{l}\text { Sindicatos u } \\
\text { organizaciones de } \\
\text { carácter laboral o } \\
\text { profesional }\end{array}$ & 696 & 2,19 & .985 & 2,34 & 1.01 \\
\hline
\end{tabular}




\begin{tabular}{|c|c|c|c|c|c|c|}
\hline Dimensión 5 & & $\begin{array}{c}\text { No ha formado parte } \\
\text { de este espacio } \\
N\end{array}$ & $\begin{array}{r}\text { E1: ha con } \\
\text { desarrollar mi } \\
X\end{array}$ & $\begin{array}{l}\text { tribuido a } \\
\text { s capacidades } \\
\text { DE }\end{array}$ & $\begin{array}{r}\text { E2: me ha } \\
\text { aplicar estas } \\
X\end{array}$ & $\begin{array}{l}\text { permitido } \\
\text { capacidades } \\
\text { DE }\end{array}$ \\
\hline \multirow{5}{*}{$\frac{O}{\mathrm{O}}$} & $\begin{array}{c}\text { Espacios virtuales y redes } \\
\text { sociales }\end{array}$ & 69 & 2,80 & .852 & 2,74 & .905 \\
\hline & $\begin{array}{l}\text { Espacios públicos abiertos } \\
\text { (calle, plaza, parque, etc) }\end{array}$ & 136 & 2,74 & .852 & 2,74 & .915 \\
\hline & $\begin{array}{l}\text { Espacios públicos cerados } \\
\text { (museos, bibliotecas, } \\
\text { centros cívicos, etc) }\end{array}$ & 143 & 2,72 & .804 & 2,63 & .833 \\
\hline & $\begin{array}{l}\text { Medios de comunicación } \\
\text { (radio, televisión, etc) }\end{array}$ & 129 & 2,59 & .842 & 2,51 & 895 \\
\hline & $\begin{array}{l}\text { Espacios recreativos } \\
\text { (bares, discotecas, etc) }\end{array}$ & 110 & 2,52 & .906 & 2,49 & .921 \\
\hline
\end{tabular}

En la Tabla 2 se observa que según los propios jóvenes el espacio considerado como más propiciador para el empoderamiento, tanto en la dimensión de desarrollo de capacidades (E1) como en la aplicación de las capacidades para actuar autónoma y consecuentemente (E2), es la familia de origen. El segundo espacio por relevancia, considerando que una cuarta parte de los jóvenes de la muestra no han tenido la experiencia de la educación superior y más de una tercera parte no han participado en grupos y asociaciones de tiempo libre, serían las relaciones de amistad actuales. Al otro extremo de la clasificación, en las últimas posiciones como espacios de empoderamiento se sitúa los partidos y organizaciones políticas, las asociaciones o confesiones religiosas, los sindicatos y organizaciones sindicales.

También destaca que las valoraciones medias de cada espacio, en las dos dimensiones, son generalmente muy parecidas, con una diferencia de menos de 0,1. Las únicas excepciones son las ONGs o asociaciones cívicas, solidarias y ecologistas; y los sindicatos: en ambos casos, la puntuación media es superior en la dimensión E2. Se han realizado correlaciones entre los espacios de empoderamiento, en las dos dimensiones (correlaciones Rho de Spearman, al ser las variables ordinales). En primer lugar, resulta destacable que casi todos los espacios están correlacionados significativamente entre sí con signo positivo; aunque la gran mayoría son de intensidad muy baja o baja.

A continuación se presentarán las correlaciones significativas ( $p<.005)$ y de intensidad por lo menos moderada (coeficientes $r_{s}>4$ ):

En la dimensión El (el desarrollo de capacidades para actuar autónomamente), se observa que algunos ítems se correlacionan más a menudo con otros. Los partidos y organizaciones de carácter político, como espacio de empoderamiento, están correlacionados con: los consejos de participación infantil y juvenil ( $\left.r_{s}=.518 ; p=.000 ; N=128\right)$; los sindicatos ( $\left.r_{s}=.501 ; p=.000 ; N=102\right)$; las asociaciones religiosas $\left(r_{s}=.400 ; p=.000 ; N=93\right)$; y las $O N G s$ $u$ otras asociaciones de carácter cívico $\left(r_{s}=.412\right.$; $p=.000 ; N=130$ ). Es decir, un joven que participe en un partido político, cuanto más desarrolla sus capacidades a través de este espacio, más aprende también a través de la participación en los otros espacios. De la misma manera, cuanto menos se empodere en los partidos, menos se empodera en los otros espacios.

Asimismo, los consejos de participación infantil y juvenil como espacio de empoderamiento en la dimensión $\mathrm{E} 1$ se correlacionan de forma más intensa con: las ONGs ( $\left.r_{s}=.447 ; p=.000 ; N=132\right)$; las asociaciones culturales ( $\left.r_{s}=.428 ; p=.000 ; N=161\right)$; las asociaciones religiosas $\left(r_{s}=.443 ; p=.000 ; N=\right.$ 93); $y$ los sindicatos ( $\left.r_{s}=.516 ; p=.000 ; N=98\right)$.

El desarrollo de capacidades (E1) a través de la participación en ONGs u otras asociaciones de carácter cívico, además de las correlaciones presentadas anteriormente, se asocia con: las escuelas de personas adultas $\left(r_{s}=.558 ; p=.000 ; N=47\right)$; los trabajos estables ( $\left.r_{s}=.409 ; p=.000 ; N=146\right)$; los sindicatos $\left(r_{s}=.549 ; p=.000 ; N=91\right) ; y$ las asociaciones religiosas $\left(r_{s}=.443 ; p=.000 ; N=112\right)$.

Se dan, además, correlaciones significativas y moderadas, entre: la educación secundaria obligatoria y la educación secundaria post-obligatoria (bachillerato y FP) $\left(r_{s}=.502 ; p=.000 ; N=750\right)$; las escuelas de personas adultas y la formación ocupacional $\left(r_{s}=.444 ; p=.000 ; N=66\right)$; las asociaciones religiosas y las escuelas de personas adultas $\left(r_{s}=.514 ; p=.000 ; N=54\right) ;$ los sindicatos $y$ las 
asociaciones religiosas $\left(r_{s}=.543 ; p=.000 ; N=78\right)$; los medios de comunicación y los espacios virtuales y redes sociales $\left(r_{\mathrm{s}}=.402 ; \mathrm{p}=.000 ; \mathrm{N}=688\right)$.

En cuanto a la dimensión E2 del empoderamiento (la aplicación de las capacidades desarrolladas para actuar autónomamente sobre la propia vida), se observa ha partir del análisis de correlaciones, que los mismos espacios tienden a correlacionarse de forma parecida a la dimensión E1. El espacio partidos y organizaciones de carácter político mantiene una correlación con: los consejos de participación infantil y juvenil $\left(r_{s}\right.$ $=.466 ; \mathrm{p}=.000 ; \mathrm{N}=125) ; \mathrm{y}$ con los sindicatos $\left(r_{s}=.439\right.$; $\mathrm{p}=.000 ; \mathrm{N}=96$ ).

Por otro lado, el empoderamiento en los consejos de participación también está correlacionado de forma moderada con el que se da en: los sindicatos ( $\left.r_{s}=.505 ; p=.000 ; N=92\right)$; en las asociaciones religiosas $\left(r_{s}=.452 ; p=.000 ; N=86\right)$; en los trabajos estables $\left(r_{s}=.431 ; p=.000 ; N=11\right) ; y$ en las escuelas de personas adultas $\left(r_{s}=.401 ; p=.005 ; N=\right.$ 47).

En el ámbito laboral, los resultados muestran que el empoderamiento que ocurre en la formación ocupacional está relacionado de manera moderada con el que se desarrolla en los trabajos temporales $\left(r_{s}=.428 ; p=.000 ; N=199\right)$ y en los trabajos estables $\left(r_{s}=.479 ; p=.000 ; N=139\right)$. Entre ellos, los trabajos temporales y los estables también se correlacionan significativamente $\left(r_{s}=.415 ; p=.000\right.$; $\mathrm{N}=310$ ).

Finalmente, los procesos de empoderamiento -como aplicación de capacidades- que se desarrollan en la educación secundaria obligatoria están correlacionados con los que se dan en la educación infantil y primaria $\left(r_{s}=.523 ; p=.000 ; N=\right.$ 790) y en la educación secundaria post-obligatoria $\left(r_{s}=.512 ; p=.000 ; N=735\right)$.

(2) Indagar en qué medida los incidentes críticos contribuyen al empoderamiento juvenil.

Seguidamente, se presentan los resultados relacionados con diferentes vivencias o incidentes críticos y cómo estos contribuyen en el desarrollo de las capacidades necesarias para actuar autónomamente sobre la propia vida (E1) y por otro lado, cómo estas vivencias permiten aplicar esas capacidades (E2).

En primer lugar, los resultados revelan que los incidentes de la lista más vividos por los jóvenes de la muestra son: conocer a una persona excepcional $(N=823)$, tener una relación afectiva o sentimental $(\mathrm{N}=822)$ y tener un éxito importante con los estudios $(\mathrm{N}=710)$. Mientras que los incidentes más excepcionales y que sólo una parte muy pequeña de los jóvenes de la muestra han experimentado son: superar una adicción ( $N=159)$, hacer pública la orientación sexual $(\mathrm{N}=208)$ y emigrar a otro país $(\mathrm{N}=217)$.

En relación al valor que atribuyen los jóvenes a cada vivencia en función de cómo esta contribuye a su empoderamiento, la primera cosa a destacar es que no hay coincidencia entre las dos dimensiones de empoderamiento E1 y E2 excepto en una de ellas. En la siguiente tabla mostramos las posiciones $(p)$ de cada vivencia para cada dimensión.

\begin{tabular}{|c|c|c|c|c|}
\hline \multicolumn{5}{|c|}{ Tabla 3: Incidentes críticos. Estadísticos descriptivos* } \\
\hline $\begin{array}{l}\text { Contribución al desarrollo de capacidades } \\
\qquad\left(E_{1}\right)\end{array}$ & E1 & $\mathrm{P}^{1}$ & E2 & $\begin{array}{l}\text { Contribución a la aplicación de capacidades } \\
\qquad(\mathrm{E} 2)\end{array}$ \\
\hline Ir a vivir sólo, con amigos o en pareja & $\begin{array}{l}3,39 \\
.873\end{array}$ & 1 & $\begin{array}{l}3,45 \\
.836\end{array}$ & Ir a vivir sólo, con amigos o en pareja \\
\hline Conocer a una persona excepcional & $\begin{array}{l}3,38 \\
.651\end{array}$ & 2 & $\begin{array}{l}3,30 \\
.989\end{array}$ & Experiencias de movilidad internacional \\
\hline Una relación afectiva o sentimental & $\begin{array}{l}3,34 \\
.715\end{array}$ & 3 & $\begin{array}{l}3,28 \\
.838\end{array}$ & Conseguir un trabajo \\
\hline La enfermedad de un ser querido & $\begin{array}{l}3,26 \\
.835\end{array}$ & 4 & $\begin{array}{l}3,23 \\
.788\end{array}$ & Una relación afectiva o sentimental \\
\hline Experiencias de movilidad internacional & $\begin{array}{l}3,25 \\
.998\end{array}$ & 5 & $\begin{array}{l}3,23 \\
.991\end{array}$ & Una ruptura familiar \\
\hline La desaparición de un ser querido & $\begin{array}{l}3,23 \\
.886\end{array}$ & 6 & $\begin{array}{l}3,18 \\
.742\end{array}$ & Conocer a una persona excepcional \\
\hline
\end{tabular}




\begin{tabular}{|c|c|c|c|c|}
\hline Una ruptura familiar & $\begin{array}{l}3,21 \\
.981\end{array}$ & 7 & $\begin{array}{c}3,18 \\
1.11\end{array}$ & Emigrar a otro país \\
\hline Conseguir un trabajo & $\begin{array}{l}3,20 \\
.825\end{array}$ & 8 & $\begin{array}{l}3,13 \\
.894\end{array}$ & Una ruptura de pareja \\
\hline Un éxito importante con los estudios & $\begin{array}{l}3,14 \\
.784\end{array}$ & 9 & $\begin{array}{l}3,11 \\
.865\end{array}$ & Un éxito importante con los estudios \\
\hline Emigrar a otro país & $\begin{array}{l}3,13 \\
1.12\end{array}$ & 10 & $\begin{array}{l}3,09 \\
.805\end{array}$ & Estar afectado por la crisis económica \\
\hline $\begin{array}{l}\text { Un éxito deportivo, cultural o artístico } \\
\text { importante }\end{array}$ & $\begin{array}{l}3,08 \\
.827\end{array}$ & 11 & $\begin{array}{l}3,09 \\
.841\end{array}$ & Un éxito profesional importante \\
\hline Una ruptura de pareja & $\begin{array}{l}3,05 \\
.886\end{array}$ & 12 & $\begin{array}{l}3,05 \\
.943\end{array}$ & La enfermedad de un ser querido \\
\hline Estar afectado por la crisis económica & $\begin{array}{l}3,01 \\
.823\end{array}$ & 13 & $\begin{array}{l}3,04 \\
1.14\end{array}$ & Ser víctima de algún abuso \\
\hline Un éxito profesional importante & $\begin{array}{l}2,99 \\
.856\end{array}$ & 14 & $\begin{array}{l}2,99 \\
1.00\end{array}$ & La desaparición de un ser querido \\
\hline Un fracaso importante en los estudios & $\begin{array}{l}2,96 \\
.947\end{array}$ & 15 & $\begin{array}{l}2,99 \\
.958\end{array}$ & Un fracaso importante en los estudios \\
\hline Ser víctima de algún abuso & $\begin{array}{l}2,87 \\
1.14\end{array}$ & 16 & $\begin{array}{l}2,93 \\
.904\end{array}$ & Un éxito deportivo, cultural o artístico importante \\
\hline $\begin{array}{l}\text { Participar en un proceso de reivindicación o de } \\
\text { movilización social o política }\end{array}$ & $\begin{array}{l}2,84 \\
.910\end{array}$ & 17 & $\begin{array}{l}2,78 \\
1.18\end{array}$ & Superar una adicción \\
\hline Superar una adicción & $\begin{array}{l}2,68 \\
1.10\end{array}$ & 18 & $\begin{array}{l}2,77 \\
1.03\end{array}$ & Quedarse sin trabajo \\
\hline $\begin{array}{l}\text { Un fracaso deportivo, cultural o artístico } \\
\text { importante }\end{array}$ & $\begin{array}{l}2,66 \\
1.04\end{array}$ & 19 & $\begin{array}{l}2,75 \\
1.05\end{array}$ & Un fracaso profesional importante \\
\hline Quedarse sin trabajo & $\begin{array}{l}2,66 \\
1.02\end{array}$ & 20 & $\begin{array}{l}2,71 \\
.987\end{array}$ & $\begin{array}{l}\text { Participar en un proceso de reivindicación o de } \\
\text { movilización social o política }\end{array}$ \\
\hline Un fracaso profesional importante & $\begin{array}{l}2,62 \\
1.02\end{array}$ & 21 & $\begin{array}{l}2,69 \\
1.17\end{array}$ & Sufrir una enfermedad grave \\
\hline Sufrir una enfermedad grave & $\begin{array}{l}2,62 \\
1.14\end{array}$ & 22 & $\begin{array}{l}2,64 \\
1.05\end{array}$ & Un fracaso deportivo, cultural o artístico importante \\
\hline Hacer pública la orientación sexual & $\begin{array}{l}2,32 \\
1.23\end{array}$ & 23 & $\begin{array}{l}2,40 \\
1.25\end{array}$ & Hacer pública la orientación sexual \\
\hline
\end{tabular}

Observamos en la tabla 3 que la vivencia a la que los jóvenes le atribuyen un valor medio más alto en relación a cuánto contribuye su empoderamiento (tanto E1 como E2), es el hecho de independizarse de la familia de origen e ir a vivir solo/a, con pareja o amigos. Sin embargo, sólo un $42,58 \%$ de los jóvenes de la muestra han podido tener dicha experiencia.
La segunda vivencia identificada por orden de valoración como más empoderadora en la dimensión 1 -desarrollar capacidades- es conocer a una persona excepcional seguida de una relación afectiva o sentimental. En cambio, las vivencias que mayor contribuyen a la segunda dimensión del empoderamiento -aplicación de capacidadesson: haber tenido una experiencia de movilidad 
internacional, aunque es destacable que sólo un $28,65 \%$ de la muestra ha tenido la oportunidad de tener dicha vivencia. La tercera posición la ocupa la experiencia de conseguir un trabajo como evento que más permite aplicar las capacidades.

Por otro lado, los fracasos profesionales, el sufrir una enfermedad o el hecho de hacer pública la propia orientación sexual son los eventos menos valorados en relación con las posibilidades que ofrecen para desarrollar capacidades (E1).

Las vivencias con las diferencias más altas a favor de la dimensión E1 son: la desaparición de una persona querida, la enfermedad de una persona querida, los éxitos deportivos, culturales o artísticos, y las relaciones afectivas y sentimentales. Es decir, estas vivencias contribuyen a unos aprendizajes relevantes (E1) en mayor medida respecto a la toma efectiva de decisiones (E2).

En cambio, tienen un nivel medio más elevado en la dimensión E2: ser víctimas de algún abuso, participar en una reivindicación política, los fracasos profesionales, quedarse sin trabajo y los éxitos profesionales.
(3) Conocer las etapas de la vida de los jóvenes en las que el empoderamiento se da con mayor intensidad.

A continuación se presentan los resultados de empoderamiento en relación con las etapas de la vida y la edad de los jóvenes en el momento de responder al cuestionario.

En primer lugar, se analizó el empoderamiento, en sus dimensiones El y E2, en función de la edad de los jóvenes que participaron en el estudio según sus respuestas de la dimensión 3 del cuestionario, donde evaluaban retrospectivamente el grado en qué habían tenido las capacidades para actuar autónomamente y el grado en qué el contexto les permitió aplicar esas capacidades para cada etapa de la vida. Se observa en la Figura 1 que la tendencia es positiva en ambas dimensiones del empoderamiento; dándose el mayor incremento entre la etapa 12-15 años y la 16-18 años (de 0.60 para el E1 y de 0.65 para el E2).

Figura 1: Evolución del empoderamiento en función de la edad

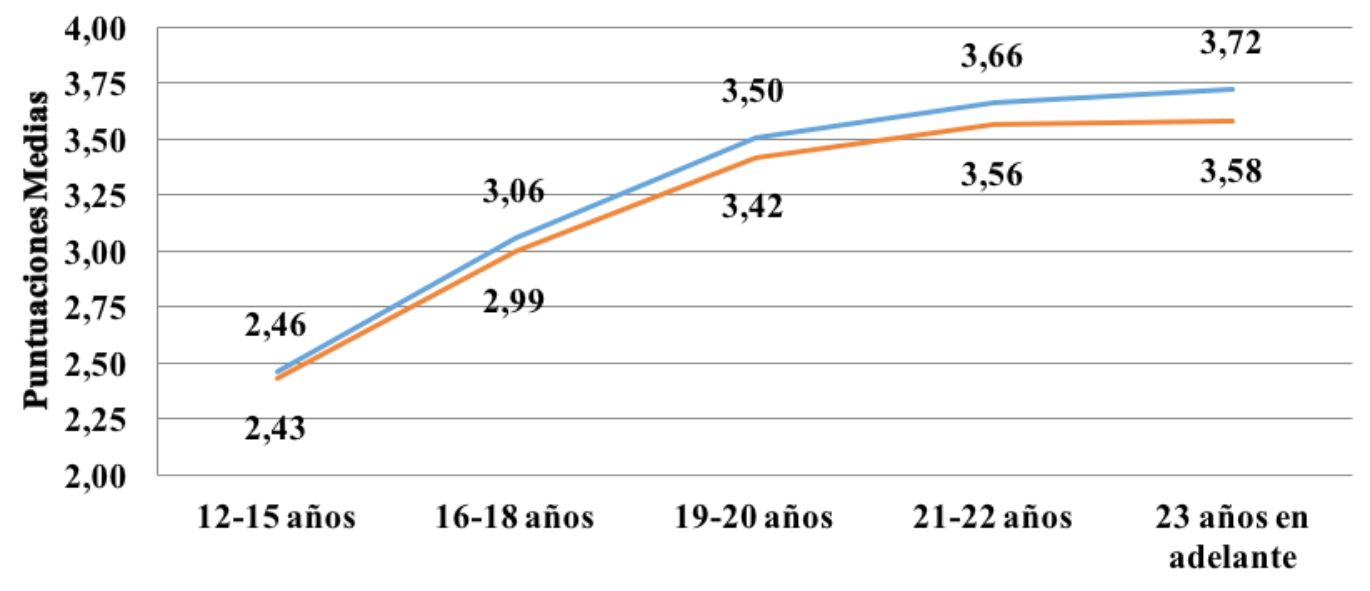

E1: desarrollo de capacidades

—E2: aplicación de capacidades para actuar autónomamente sobre mi vida

Sin embargo, al considerar las variables de nivel de empoderamiento actual en relación con la edad de los jóvenes participantes (de 19 a 25), no parece haber una relación tan clara entre edad y empoderamiento. Como se presenta en la Figura 2, ambas dimensiones parecen tener un descenso a los 22 años, mientras que vuelven a incrementar a los 23 años. 
Figura 2: Evolución del empoderamiento en función de la edad, de los 19 a los 25 años

\begin{tabular}{|c|c|c|c|c|c|c|c|}
\hline \multirow{2}{*}{$\begin{array}{l}4,00 \\
3,75\end{array}$} & \multirow[b]{2}{*}{3,58} & \multirow{2}{*}{3,68} & \multirow[b]{2}{*}{3,66} & \multirow[b]{2}{*}{3,61} & \multirow[t]{2}{*}{3,75} & \multirow[t]{2}{*}{3,84} & \multirow[t]{2}{*}{3,82} \\
\hline & & & & & & & \\
\hline & & & & & & & 3,74 \\
\hline \multirow{2}{*}{$\begin{array}{l}3,25 \\
3,00\end{array}$} & 3,63 & 3,63 & 3,58 & 3,52 & 3,59 & 3,64 & \\
\hline & & & & & & & \\
\hline \multirow{2}{*}{$\begin{array}{l}2,75 \\
2,50\end{array}$} & & & & & & & \\
\hline & & & & & & & \\
\hline 2,25 & & & & & & & \\
\hline 2,25 & & & & & & & \\
\hline 2,00 & 19 años & 20 años & 21 años & 22 años & 23 años & 24 años & 25 años \\
\hline
\end{tabular}

\section{- E1: desarrollo de capacidades}

\section{E2: aplicación de capacidades para actuar autónomamente sobre mi vida}

Las pruebas de correlaciones (coeficiente rho de Spearman, al no cumplir los supuestos para pruebas paramétricas) revelaron una correlación débil entre la dimensión $\mathrm{E} 1$ del empoderamiento actual y la edad $r_{s}(754)=.118, p=.001$; y la relación entre la dimensión E2 y la edad no resultó significativa, $r_{s}(739)=.040, p=.279$. Para profundizar en esta relación entre la edad y el empoderamiento, se realizaron pruebas de chi cuadrado asociando las variables de empoderamiento actual (E1 y E2) y la edad actual. Respecto a la dimensión El, la prueba de chi cuadrado revela que los porcentajes de puntuaciones 1, 2, 3 y 4 en empoderamiento están significativamente asociados a la edad, $X^{2}$ $(18, N=756)=32.88, p=.017$. En la Tabla 4 se puede observar que hay un progresivo incremento de puntuaciones máximas, según aumenta la edad de los jóvenes de la muestra.

\begin{tabular}{|c|c|c|c|c|}
\hline \multicolumn{5}{|c|}{$\begin{array}{c}\text { Tabla 4: Tabla de contingencia: } \\
\text { nivel de empoderamiento (E1) y edad }\end{array}$} \\
\hline & \multicolumn{4}{|c|}{$\begin{array}{r}\text { Nivel de capacidades para actuar } \\
\text { autónomamente (E1)* }\end{array}$} \\
\cline { 2 - 5 } & 1 & 2 & 3 & 4 \\
\hline 19 años & $5.0 \%$ & $.0 \%$ & $27.5 \%$ & $67.5 \%$ \\
\hline 20 años & $1.0 \%$ & $1.5 \%$ & $26.1 \%$ & $71.4 \%$ \\
\hline 21 años & $1.1 \%$ & $2.2 \%$ & $26.1 \%$ & $70.7 \%$ \\
\hline 22 años & $4.7 \%$ & $1.4 \%$ & $22.3 \%$ & $71.6 \%$ \\
\hline 23 años & $0.9 \%$ & $3.8 \%$ & $14.2 \%$ & $81.1 \%$ \\
\hline 24 años & $0 \%$ & $.0 \%$ & $16.2 \%$ & $83.8 \%$ \\
\hline 25 años & $0 \%$ & $2.0 \%$ & $14.0 \%$ & $84.0 \%$ \\
\hline *1. Nada 2. Poco 3. Bastante 4. Mucho. & \\
\hline \multicolumn{5}{|c|}{$0 \%$} \\
\hline
\end{tabular}

El análisis de residuos de Haberman (1973) indica que los jóvenes de 24 años que obtienen un 4 en el nivel de empoderamiento (E1) son más de los casos esperados, si las dos variables fueran independientes (residuo tipificado corregido de 2.3); lo que indica que hay una asociación significativa entre edad y las capacidades necesarias para actuar autónomamente (E1)", aunque puede que no sea lineal; y que en esta progresión, el incremento más significativo se da a los 24 años.

Respecto a la dimensión E2, aunque se observa una progresión en el nivel de empoderamiento especialmente entre los 22 y los 25 años (Tabla 5), la prueba de chi cuadrado no revela una asociación significativa entre edad y empoderamiento, $\left.X^{2} 18, N=741\right)=16.63, p=.549$.

\begin{tabular}{|c|c|c|c|c|}
\hline \multicolumn{5}{|c|}{ Tabla 5: Tabla de contingencia: } \\
nivel de empoderamiento (E2) y edad \\
\hline & \multicolumn{4}{|c|}{$\begin{array}{r}\text { Nivel de aplicación de capacidades para } \\
\text { actuar autónomamente (E2) }\end{array}$} \\
\hline 19 años & $0 \%$ & $3.7 \%$ & $29.6 \%$ & $66.7 \%$ \\
\hline 20 años & $1.0 \%$ & $4.0 \%$ & $26.1 \%$ & $68.8 \%$ \\
\hline 21 años & $1.1 \%$ & $6.5 \%$ & $26.1 \%$ & $66.3 \%$ \\
\hline 22 años & $4.0 \%$ & $3.4 \%$ & $29.5 \%$ & $63.1 \%$ \\
\hline 23 años & $3.7 \%$ & $4.7 \%$ & $20.6 \%$ & $71.0 \%$ \\
\hline 24 años & $0.9 \%$ & $6.0 \%$ & $21.4 \%$ & $71.8 \%$ \\
\hline 25 años & $2.0 \%$ & $2.0 \%$ & $16.0 \%$ & $80.0 \%$ \\
\hline * 1. Nada 2. Poco 3. Bastante 4. Mucho. & & \\
\hline
\end{tabular}


Para explicar el resultado del nivel de empoderamiento alto (E1) en los jóvenes de 24 años, se han explorado las características típicas de los jóvenes de esta edad en función de la variable trabajo, ya que ésta se reveló como una de las más características de los jóvenes con niveles elevados de empoderamiento en estudios anteriores (Pineda-Herrero et al., en revisión). Se observa que el $64.2 \%$ de los chicos y chicas de esta edad trabajan, siendo este el porcentaje más elevado de todas las franjas de edad. La distribución de jóvenes que trabajan y no trabajan es significativamente diferente por edad, $\left.X^{2} 6, N=774\right)=55.04$, $p=.000$, y el residuo tipificado corregido en esa celda es de 4.9. También es remarcable que, para el $38 \%$ de los jóvenes de esa edad, los ingresos del trabajo les permiten independencia económica de sus familias; siendo esta la asociación que contribuye en mayor media (con un residuo tipificado corregido de 4.7) al chi cuadrado, $X^{2}(6, N=$ 885) $=61.06, p=.000$.

\section{Discusión y conclusiones}

El estudio tenía como finalidad aportar datos sobre el empoderamiento juvenil, obtenidos desde la propia percepción de los jóvenes que contribuyeran a una reflexión sobre los espacios, los momentos y las vivencias que son parte del proceso de empoderamiento. Por un lado, una de las aportaciones de este trabajo ha sido romper con el sesgo adulto-céntrico en el estudio del empoderamiento juvenil que denunciaban autores como Kaplan et al. (2009). Por otro lado, se han presentado las auto-percepciones de los jóvenes en las dos dimensiones constitutivas del empoderamiento (Úcar et al., 2016): el desarrollo de capacidades y la aplicación de estas capacidades para tomar decisiones y actuar autónomamente sobre la propia trayectoria vital; lo que permite analizar más en detalle el rol de los espacios y los eventos, en el proceso de empoderamiento.

En relación a la identificación de espacios que propician el empoderamiento, en primer lugar hemos constatado que los contextos más próximos como la familia de origen y las relaciones de amistad actuales son los más valorados por los jóvenes, como apuntaban Mc Hale, Dotterer y Kim (2009) y Masa (2009). A su vez, aquellos jóvenes que han tenido la oportunidad de frecuentar espacios tales como las asociaciones de tiempo libre, les atribuyen un elevado impacto en su empoderamiento, casi tanto como la propia familia.

En segundo lugar, se identifican espacios que favorecen en mayor medida una dimensión del empoderamiento u otra. Por ejemplo, destaca que la participación en ONGs o en otro tipo de asociaciones de carácter cívico, solidario o ecologista contribuye en mayor medida a la aplicación de capacidades para la actuación autónoma; así como la participación en sindicatos $u$ otras organizaciones laborales o profesionales.

Los resultados nos permiten constatar el valor de los espacios relacionales, laborales y de participación, más allá de los espacios de educación formal, en los procesos de empoderamiento; y la importancia de potenciar la participación en espacios de ocio como las asociaciones deportivas, los grupos de educación en el tiempo libre y las ONGs para favorecer la toma de decisiones autónomas y conscientes.

Los resultados también permiten comprobar que existe relación entre todos los espacios, sobre todo los que están incluidos en la dimensión de participación ciudadana; tanto para la dimensión de desarrollo de capacidades, como para la aplicación de éstas. Esto sugiere que la participación en espacios políticos y sociales (asociaciones culturales, ONGs, consejos de participación infantil y juvenil, partidos, asociaciones religiosas, sindicatos), realza la experiencia en otros espacios y dimensiones del empoderamiento. Las capacidades que se desarrollan y las competencias que se adquieren en los espacios de participación social y política son valoradas significativamente tanto por su dimensión formativa (no hay que olvidar la oportunidad de formación cívica y política que estos contextos ofrecen), como también por la posibilidad de poder llevar a cabo proyectos concretos que permiten a estos jóvenes tomar decisiones autónomamente, ensayar y aplicar dichas capacidades. Estos marcos no formales se convierten en este caso en auténticas escuelas de ciudadanía y laboratorios de responsabilidad social.

En relación a las experiencias vitales y su contribución al empoderamiento hemos comprobado que emanciparse (ir a vivir solo, con amigos o en pareja) o tener una experiencia internacional son las vivencias consideradas por los jóvenes como las más empoderadoras. Ambas experiencias, debido al contexto socio-económico actual suponen un gran reto y tal y como apuntaban Jenning et al (2008) la creación de oportunidades y el planteamiento de retos son grandes estrategias de empoderamiento.

Según el informe del Observatorio catalán de la juventud (2016) sobre la situación laboral de las personas jóvenes en Cataluña, la tasa de emancipación de los jóvenes entre 16 y 29 años es del $23.7 \%$, lo cual supone un retraso en la edad de emancipación respecto al resto de países europeos. La emancipación de los jóvenes se ha visto afectada por las condiciones del mercado laboral. 
En relación a las experiencias de movilidad internacional, concebidas como altamente empoderadoras según los jóvenes también se han visto afectadas por la reducción de ayudas, las cuales han bajado a la mitad los jóvenes estudiantes que realizan estancias en el extranjero (por ejemplo, la partida para estudiar en el extranjero ha caído un $71 \%$ desde 2011 hasta la actualidad - el país $4 / 11 / 2013$ )

Es destacable que las experiencias vitales que requieren una actitud de superación personal, como la enfermedad o la desaparición de un ser querido, una ruptura familiar o de pareja y la emigración a otro país, son reconocidas por los jóvenes como una vivencia que les permitió realizar aprendizajes relevantes y tomar decisiones importantes sobre su vida. En este caso, el empoderamiento se manifiesta como un proceso intransferible que debe realizar cada uno (Fetterman y Wandersman, 2007), mediante la atribución de significado y la forma de relacionarse con los diferentes acontecimientos.

Por otro lado, entre las vivencias menos valoradas por los jóvenes encuestados como fuente de empoderamiento, se encuentran los fracasos profesionales, deportivos, culturales o artísticos, el quedarse sin trabajo, el sufrir una enfermedad grave y hacer pública la propia orientación sexual; lo cual sugiere la necesidad de profundizar en los trabajos sobre la resiliencia de los jóvenes y su capacidad de superación como factor de empoderamiento (Omar et al. 2011).

Por último, en cuanto a las etapas vitales y su relación con el empoderamiento, hemos comprobado que se da una evolución progresiva entre los 19 y los 25 años, no siempre lineal, y que el incremento más significativo en el nivel de empoderamiento $\mathrm{E}_{1}$ (el desarrollo de capacidades) se da en la etapa vital de los 24 años; lo que se puede explicar por la mayor inserción, en esta franja de edad, en el trabajo y su consecuente independencia económica de las familias de origen.

En cambio, la aplicación de capacidades para actuar autónomamente no varía significativamente en función de la edad después de los 19 años. Podemos interpretar que la formación no es suficiente por si sola para garantizar el empoderamiento, pues es necesario que el contexto permita y facilite la aplicación de las capacidades adquiridas. La educación (tanto la formal, como la no formal y la informal) aumentan con la edad y con ella la dimensión primera del empoderamiento (E1), es decir el desarrollo de capacidades para actuar autónomamente. Pero si el contexto no permite la aplicación de estas capacidades (E2) difícilmente podemos hablar de empoderamiento real (Soler et al. 2017).

En definitiva, el desarrollo de las capacidades necesarias para actuar autónomamente sí está condicionado por la edad (la experiencia y la formación que esta conlleva), en cambio el grado en que el contexto permite aplicar estas capacidades no tiene una relación clara con la edad.

Este estudio nos sirve como punto de partida para seguir desarrollando el conocimiento sobre los procesos de empoderamiento en jóvenes, en función de los espacios, momentos y etapas vitales analizadas.

\section{Referencias bibliográficas}

Blanchet-Cohen, N., \& Brunson, L. (2014). Creating Settings for Youth Empowerment and Leadership: An Ecological Perspective, Child \& Youth Services, 35 (3), 216-236, DOI: 10.1080/0145935X.2014.938735.

Boluijt, B., \& de Graaf, L. J. (2010). Preaching empowerment, practicing participation: The use of empowerment and citizen participation in Dutch local democracies. Onbekend: Tilburgse School voor Politiek en Bestuur (TSPB).

Fetterman, D. M., \& Wandersman, A. (2007). Empowerment Evaluation: Yesterday, Today, and Tomorrow. American Journal of Evaluation 28 (2), 179-98. doi:10.1177/1098214007301350.

Hennink, M., Kiiti, N., Pillinger, M., \& Jayakaran, R. (2012). Defining empowerment: perspectives from international development organisations. Development in Practice, 22 (2), 202-215. Holte-McKenzie, M.; Forde, S. \& Theobald, S. (2006). Development of a Participatory Monitoring and Evaluation Strategy. Evaluation and program planning, 29 (4), 365-76.

Jennings, L. B., Parra-Medina, D. M., Hilfinger Messias, D. K., \& McLoughlin, K. (2008). Toward a critical social theory of youth empowerment. Journal of Community Practice, 14, 31-55.

Kaplan, S. J., L. Skolnik, L., \& Turnbull, A. (2009). Enhancing the Empowerment of Youth in Foster Care: Supportive Services. Child Welfare, 88 (1), 133-161.

Kelly, C., \& Carson, E. (2012). The Youth Activist Forum: Forging a Rare, Disability-Positive Space That Empowers Youth. Journal of Youth Studies 15 (8), 1089-1106. doi:10.1080/13676261.2012.693595.

Masa, M. (2009). Adolescentes en Euskadi. Una aproximación desde el empoderamiento. Vitoria-Gasteiz: Emakunde. Instituto Vasco de la Mujer. 
Mchale, S. M., Dotterer, A., \& Kim, J.-Y. (2009). An ecological perspective on the Media and Youth Development. American Behavioral Scientist, 52 (8), 1186-1203.

Morrissey, J. (2000). Indicators of Citizen Participation: Lessons from Learning Teams in Rural EZ/EC Communities. Community Development Journal, 35 (1), 59-74.

Morton, M. H., \& Montgomery, P. (2013). Youth Empowerment Programs for Improving Adolescents' Self-Efficacy and Self-Esteem: A Systematic Review. Research on Social Work Practice, 23 (1), 22-33.

Mosedale, S. (2003). "Towards a framework for assessing empowerment". Paper presented at the Conference New Directions in Impact Assessment for Development. Methods and Practice, Manchester, November 24-25.

Nolas, S-M. (2014). Exploring Young People's and Youth Workers' Experiences of Spaces for 'youth Development': Creating Cultures of Participation. Journal of Youth Studies, 17 (1), 26-41. Doi:10.1080/13676261.2013.793789.

Observatori Català de la Joventut. (2016). Situació laboral de les persones joves a Catalunya. Generalitat de Catalunya. Departament de Treball, Afers Socials i Famílies. Direcció General de Joventut.

Omar, A., Paris, L., Delgado, H. U., Ameida da Silva, S. E., \& Aguiar de Souza, M. (2011). Un modelo explicativo de resiliencia en jóvenes y adolescentes. Psicologia Em Estudo, 16 (2), 269-277.

Pearrow, M. M. (2011). Opportunity for Unity Collaborative: Building Healthy Communities by Empowering Young Adults. Community Development 42 (2), 167-80.

Peterson, N. A. (2014). Empowerment theory: clarifying the nature of higher-order multidimensional constructs. American Journal of Community Psychology 53 (1-2), 96-108.

Peterson, N. A., Peterson, C. H., Agre, L., Christens, B. D., \& Morton, C. M. (2011). Measuring youth empowerment: validation of a Sociopolitical Control Scale for Youth in an urban community context. Journal of Community Psychology, 39 (5): 592-605. Doi:10.1002/jcop.20456.

Pick, S., Sirkin, J., Ortega, I., Osorio, P., Martínez, R., Xocolotzin, U., \& Givaudan, M. (2007). Escala Para Medir Agencia Personal y Empoderamiento (ESAGE). Revista Interamericana de Psicología, 41 (3), 295-304.

Pineda-Herrro, P., Agud-Morell, I., Ciraso-Cali, A., \& Soler-Masó, P. (en revisión). In their own words: Elements of Youth Empowerment in Spain. Journal of Youth Studies.

Pineda-Herrero, P., Agud-Morell, I., \& Núñez, H. (2015). Empoderamiento juvenil: conceptos y significados del empoderamiento para sus protagonistas. IV Congreso Iberoamericano de Pedagogía Social. Ciudad de Puebla, México.

Russell, S. T., Muraco, A., Subramaniam, A., \& Laub, C. (2009). Youth empowerment and high school gay-straight alliances. Journal of Youth and Adolescence, 38 (7), 891-903. Doi:10.1007/s10964-008-9382-8.

Sathar, Z.A., \& Kazi, S. 1997. Women's Autonomy, Livelihood \& Fertility: A Study of Rural Punjab. Islamabad: Instituto Pakistan Institute of Development Economics.

Soler, P., Trilla, J., Jiménez-Morales, M., \& Úcar, X. (2017). La construcción de un modelo educativo del empoderamiento juvenil: espacios, momentos y procesos, Pedagogía Social. Revista Interuniverstiaria, 30, 25-40. DOI:10.7179/ PSRI_2017.30.02.

Stolte, L. C., Isenbarger, M., \& Cohen, A. K. (2014). Measuring Civic Engagement Processes and Youth Civic Empowerment in the Classroom: The CIVVICS Observation Tool, The Clearing House: A Journal of Educational Strategies, Issues and Ideas, 87:1, 44-51, DOI: 10.1080/00098655.2013.842531.

Tromp, M. (2007). A system of empowerment indicators for a corporate work environment. Johannesburg: University of Johannesburg.

Úcar Martínez, X., Jiménez-Morales, M., Soler Masó, P., \& Trilla, J. (2016). Exploring the conceptualitzation and research of empowerment in the field of youth. International Journal of Adolescence and Youth. Doi: http://dx.doi.org/10.10 80/02673843.2016.1209120

Wagaman, M. A. (2011). Social Empathy as a Framework for Adolescent Empowerment. Journal of Social Service Research 37 (3), 278-293.

Wright, K. (2010). “Giving Voice' to Youths in Out-of-Home Placements. Journal of Human Behavior in the Social Environment, 20 (3), 335-348. 


\section{CÓMO CITAR ESTE ARTÍ́CULO}

Agud-Morell, I., Ciraso-Calí, A., Pineda-Herrero, P., \& Soler-Masó, P. (2017). Percepción de los jóvenes sobre los espacios y momentos en su proceso de empoderamiento. Una aproximación cuantitativa. Pedagogía Social. Revista Interuniversitaria, 30, 51-66. DOI:10.7179/PSRI_2017.30.04.

\section{DIRECCIÓN COMPLETA DE LOS AUTORES}

Ingrid Agud-Morell: Departamento Pedagogía Sistemática y Social. Universidad Autónoma de Barcelona. ingrid.agud@uab.cat

Anna Ciraso-Calí: Departamento Pedagogía Sistemática y Social. Universidad Autónoma de Barcelona. annaciraso@gmail.com

Pilar Pineda-Herrero: Departamento Pedagogía Sistemática y Social. Universidad Autónoma de Barcelona. pilar.pineda@uab.cat

Pere Soler-Masó: Departamento de Pedagogía. Universidad de Girona. pere.soler@udg.edu

\section{PERFIL ACADÉMICO}

Ingrid Agud-Morell: Profesora lectora en el Departamento de Pedagogía Sistemática y Social de la Universitat Autònoma de Barcelona. Doctora en Educación y Sociedad por la Universidad de Barcelona. Su investigación se centra principalmente en la participación política, social e institucional de la infancia y la adolescencia; la educación en valores y los derechos de la infancia. Miembro del Grupo de Investigación en Educación Moral (GREM, UB) y colaboradora del Grupo de Investigación en Políticas Educativas (GIPE, UAB). Web: http://orcid.org/o000-0003-3759-5784.

Anna Ciraso-Calí: Pedagoga e investigadora, vinculada al Grupo Interdisciplinar de Políticas Educativas (GIPE-UAB) y al Grupo de investigación EFI (Eficacia de la Formación). Su actividad profesional se ha desarrollado en la educación no formal y en la formación continua. Como investigadora, participa en proyectos sobre evaluación de la transferencia, formación continua del profesorado, formación a centros de trabajo, evaluación participativa y empoderamiento.

Pilar Pineda-Herrero: Profesora titular de Economía de la Educación y Pedagogía Laboral en el Departamento de Pedagogía Sistemática y Social de la Universidad Autónoma de Barcelona. Sus líneas de trabajo son la educación y el trabajo, la formación continua y su eficacia, la inserción laboral de los jóvenes, y el desarrollo comunitario. Dirige el grupo de investigación EFI -Eficacia de la Formación, http://grupsderecerca.uab.cat/efi/es-. Forma parte del grupo de investigación GIPE -Grupo Interdisciplinar de Políticas Educativas, http://gipe-igep.org/-. Ha dirigido numerosos estudios sobre evaluación y eficacia de la formación para diversos colectivos y sectores: administración pública, profesorado, personal sanitario, industria, empresas de servicios, fundaciones educativas, etc. Ha publicado diversos libros y artículos sobre sus líneas de investigación en revistas científicas nacionales e internacionales, que pueden consultarse en los links anteriores. 
Pere Soler-Masó: Profesor titular de universidad en el Departamento de Pedagogía en la Universidad de Girona. Su actividad docente y de investigación se centra en las políticas de juventud, el asociacionismo y la participación infantil y juvenil, el desarrollo comunitario, la educación en el tiempo libre y la animación sociocultural. Actualmente es miembro del Grupo de Investigación en Políticas, Programas y Servicios Educativos y Socioculturales (GRES) del Instituto de Investigación Educativa - UdG y del Grupo de Investigación Consolidado (SGR) en Educación, participación e inclusión (GREPI) (2014SGR 774- GRC). Es coordinador general desde el 2007 del Máster Oficial Interuniversitario en Juventud y Sociedad (MIJS) impartido por seis universidades públicas. Pueden consultarse algunas de sus publicaciones en el siguiente enlace: http://girona. academia.edu/PereSolerMasó. 